\title{
The resonant damping of oscillations of coronal loops with elliptic cross-sections
}

\author{
M. S. Ruderman ${ }^{\star}$ \\ Department of Applied Mathematics, University of Sheffield, Hicks Building, Hounsfield Road, Sheffield S3 7RH, UK
}

Received 16 April 2003 / Accepted 7 July 2003

\begin{abstract}
Motivated by recent Transition Region and Coronal Explorer (TRACE) observations of damped oscillations in coronal loops, Ruderman \& Roberts (2002), studied resonant damping of kink oscillations of thin straight magnetic tubes in a cold plasma. In their analysis, Ruderman \& Roberts considered magnetic tubes with circular cross-sections. We extend their analysis for magnetic tubes with elliptic cross-sections. We find that there are two infinite sequences of the eigenfrequencies of the tube oscillations, $\left\{\omega_{n c}\right\}$ and $\left\{\omega_{n s}\right\}, n=1,2, \ldots$. The eigenfrequencies $\left\{\omega_{n c}\right\}$ and $\left\{\omega_{n s}\right\}$ correspond to modes with $2 n$ nodes at the tube boundary. In particular, $\omega_{1 c}$ and $\omega_{1 s}$ correspond to two kink modes. These modes are linearly polarized in the direction of the large and small axis of the tube elliptic cross-section respectively. The sequence $\left\{\omega_{n c}\right\}$ is monotonically growing and $\left\{\omega_{n s}\right\}$ monotonically decreasing, and they both tend to $\omega_{k}$ as $n \rightarrow \infty$, where $\omega_{k}$ is the frequency of the kink mode of tubes with circular cross-sections. In particular, $\omega_{1 c}<\omega_{k}<\omega_{1 s}$. We calculate the decrements of the two kink modes and show that they are of the order of decrement of the kink mode of a tube with a circular cross-section.
\end{abstract}

Key words. magnethohydrodynamics (MHD) - plasmas - Sun: corona - waves

\section{Introduction}

This study was motivated by the recent TRACE observations of damped coronal loop oscillations (Aschwanden et al. 1999; Nakariakov et al. 1999; Schrijver \& Brown 2000; Aschwanden et al. 2002; Schrijver et al. 2002). Aschwanden et al. (1999) and Nakariakov et al. (1999) interpreted these observations in terms of the kink mode of oscillation of a coronal loop. Nakariakov et al. (1999) noted that the loop oscillations were strongly damped with the characteristic damping time equal to a few periods of oscillations.

Different mechanisms of damping of coronal loop oscillations were discussed by Roberts (2000) and Ofman \& Aschwanden (2002). Ruderman \& Roberts (2002) suggested that the damping of these oscillations is due to resonant absorption, which is the energy transfer from the global mode of oscillation of a coronal loop into quasi-Alfvénic oscillations in a thin dissipative layer. This layer embraces an ideal resonant position where the frequency of the global mode matches the local Alfvén frequency. To study this process Ruderman \& Roberts (2002) solved the initial value problem for a thin straight magnetic tube with a circular cross-section and a thin inhomogeneous layer at its boundary. They showed that a damped kink oscillation of this tube emerges from an arbitrary initial perturbation after the time of order of a few periods of this

^ e-mail: M.S.Ruderman@sheffield.ac.uk oscillation. The characteristic damping time is of order of the oscillation period times the ratio of the tube radius and the thickness of the inhomogeneous layer. Goossens et al. (2002) used the expression for the resonant damping rate of kink oscillations to investigate damped coronal loop oscillations in a sample of 11 loops provided by Aschwanden et al. (2002).

One of the main results of Ruderman \& Roberts (2002) is that the asymptotic state attained by an initial perturbation after a few periods of the tube kink oscillation is completely determined by the properties of the damped kink eigenmode of the tube. This result can be easily generalized for configurations more complicated than a straight magnetic tube with a circular cross-section in a cold unstratified plasma considered by Ruderman \& Roberts (2002). Hence, it is not necessary to solve the initial value problem every time to study the asymptotic state of tube oscillation caused by an initial perturbation. Rather it is enough to study the damped eignemodes of the tube oscillations. This observation enables us to concentrate on studying eigenmodes in this paper. The aim of the paper is to study the damped eigenmodes of a thin magnetic tube with an elliptic cross-section in a cold plasma. Similar to Ruderman \& Roberts (2002), we assume that the damping of oscillations is due to resonant absorption. This absorption occurs in a dissipative layer embracing an ideal resonant line inside a thin inhomogeneous layer at the tube boundary. In the next section we formulate the problem. In Sect. 3 we derive the equations 


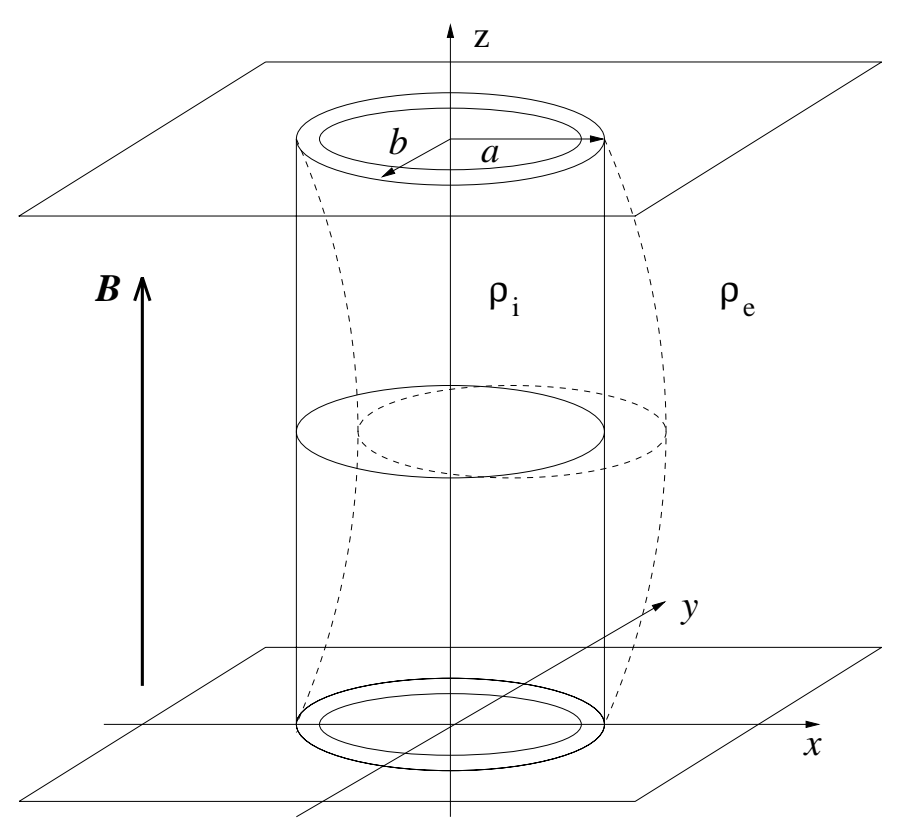

Fig. 1. A sketch of the equilibrium state, showing a magnetic flux tube with plasma density $\rho_{\mathrm{i}}$ embedded in a plasma with density $\rho_{\mathrm{e}}$. The equilibrium magnetic field is everywhere has strength $B$. The equilibrium density varies in the thin layer from $\rho_{\mathrm{i}}$ to $\rho_{\mathrm{e}}$. The dashed lines show the perturbed magnetic tube in its kink mode of oscillation.

relating the pressure perturbation at the boundaries of the inhomogeneous layer. In Sect. 4 we study undamped waves in a homogeneous tube and obtain the dispersion relations for these waves in the approximation of an infinitely thin tube. In Sect. 5 we investigate resonant damping of kink modes. We present the summary of our results and their discussion in Sect. 6.

\section{Formulation}

We consider a magnetic tube in a cold ideal plasma. To describe plasma motions we use the linear system of ideal MHD equations

$\rho \frac{\partial \boldsymbol{v}}{\partial t}=\frac{1}{\mu}(\nabla \times \boldsymbol{b}) \times \boldsymbol{B}, \quad \frac{\partial \boldsymbol{b}}{\partial t}=\nabla \times(\boldsymbol{v} \times \boldsymbol{B})$.

Here $\boldsymbol{v}$ is the velocity, $\rho$ the equilibrium density, $\boldsymbol{B}$ the equilibrium magnetic field, and $\boldsymbol{b}$ the perturbation of the magnetic field; $\mu$ is magnetic permeability of empty space.

The equilibrium configuration is a straight magnetic tube (see Fig. 1). The equilibrium magnetic field is everywhere along the $z$-direction of Cartesian coordinates $x, y, z$, and it also has everywhere the same magnitude $B$. The equilibrium density is equal to $\rho_{\mathrm{i}}$ inside the tube, and $\rho_{\mathrm{e}}$ outside the tube, $\rho_{\mathrm{e}}<\rho_{\mathrm{i}}$. It monotonically increases from $\rho_{\mathrm{e}}$ to $\rho_{\mathrm{i}}$ in a thin layer at the tube boundary.

In what follows we assume that the tube has as elliptic cross-section. We introduce elliptic coordinates $s$ and $\varphi$ in the $x y$-plane (see Fig. 2). These coordinates are related to Cartesian cordinates by

$x=\sigma \cosh s \cos \varphi, \quad y=\sigma \sinh s \sin \varphi$,

where $\sigma$ is a quantity with the dimension of length. We assume that the equilibrium density depends on $s$ only, $\rho=\rho(s)$.

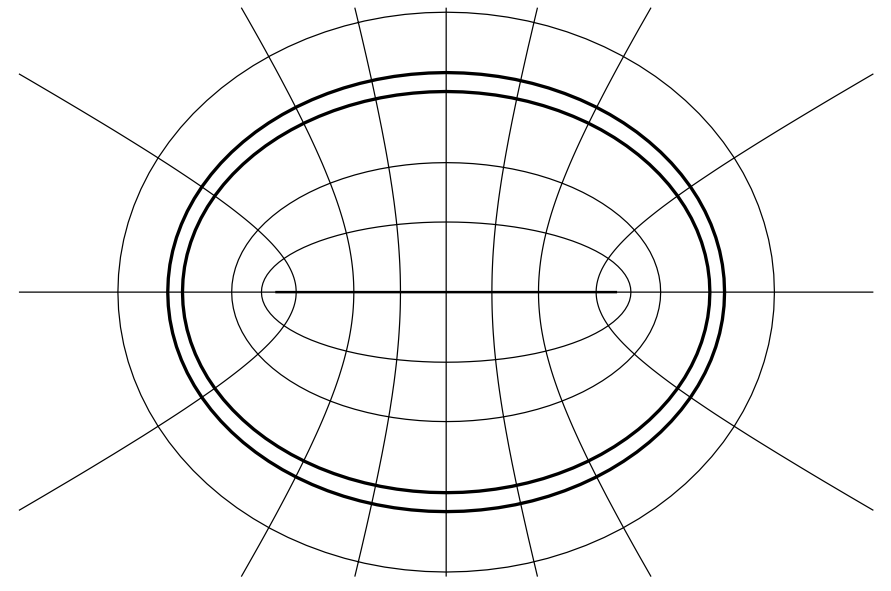

Fig. 2. A sketch of elliptic coordinates in the $x y$-plane. The ellipses are the $\varphi$ coordinate lines, and the hyperbolas are the $s$ coordinate lines. The two thick ellipses are the boundaries of the inhomogeneous layer.

The equation of the tube boundary is $s=s_{0}$. Hence, the tube cross-section is an ellipse with the large and small half-axis, $a$ and $b$, given by

$a=\sigma \cosh s_{0}, \quad b=\sigma \sinh s_{0}$.

The ellipse eccentricity is $\varepsilon=1 / \cosh s_{0}$. The density monotonically increases from $\rho_{\mathrm{e}}$ to $\rho_{\mathrm{i}}$ in a thin layer $s_{0}-\delta \leq s \leq s_{0}$, where $\delta \ll s_{0}$. The thickness of this layer increases from $b \delta$ in the $x$-direction $(\varphi=0)$ to $a \delta$ in the $y$-direction $(\varphi=\pi / 2)$. The equilibrium density remains constant and equal to $\rho_{\mathrm{i}}$ for $s \leq s_{0}-\delta$.

Eliminating $b_{x}$ and $b_{y}$ from (1), we obtain

$\frac{\partial^{2} \boldsymbol{v}}{\partial t^{2}}-V_{\mathrm{A}}^{2} \frac{\partial^{2} \boldsymbol{v}}{\partial z^{2}}=-\frac{1}{\rho} \nabla_{\perp} \frac{\partial P}{\partial t}, \quad \frac{\partial P}{\partial t}=-\rho V_{\mathrm{A}}^{2} \nabla_{\perp} \cdot \boldsymbol{v}$.

Here $P, V_{\mathrm{A}}^{2}$ and $\nabla_{\perp}$ are the perturbation of the magnetic pressure, the square of the Alfvén speed, and the perpendicular gradient given by

$P=\frac{B b_{z}}{\mu}, \quad V_{\mathrm{A}}^{2}=\frac{B^{2}}{\mu \rho}, \quad \nabla_{\perp}=\nabla-\boldsymbol{e}_{z} \frac{\partial}{\partial z}$,

where $b_{z}$ is the $z$-component of the perturbation of the magnetic field and $\boldsymbol{e}_{z}$ is the unit vector in the $z$-direction.

We assume that the magnetic tube is bounded at $z=0$ and $z=L$ by dense ideal infinitely conducting plasmas with the magnetic field frozen in these plasmas. This implies the boundary conditions

$\boldsymbol{v}=0 \quad$ at $\quad z=0, L$.

Equation (6) coupled with the second equation in (4) implies that

$P=0 \quad$ at $\quad z=0, L$.

We consider only the fundamental mode of the tube oscillation with respect to $z$. Then it follows from (6) and (7) that $v$ and $P$ are proportional to $\sin (\pi z / L)$. Our analysis remains applicable to an overtone if we regard $L$ as the distance between successive nodes along the loop. We also restrict our analysis to the normal 
modes and take $v$ and $P$ proportional to $\mathrm{e}^{-i \omega t}$. Then Eqs. (4) reduce to

$\rho\left(\omega^{2}-\omega_{\mathrm{A}}^{2}\right) \boldsymbol{v}=-i \omega \nabla_{\perp} P, \quad i \omega P=\rho V_{\mathrm{A}}^{2} \nabla_{\perp} \cdot \boldsymbol{v}$,

where $\omega_{\mathrm{A}}=\pi V_{\mathrm{A}} / L$ is the local Alfvén frequency.

Equations (8) are used in what follows for studying the damped oscillations of a magnetic tube.

\section{Equations for boundary values of pressure perturbation}

In this section we derive the equations relating the pressure perturbation at the boundaries of the inhomogeneous layer. We do this by steps. First we obtain the solution in the inhomogeneous layer $\left(s_{0}-\delta<s<s_{0}\right)$. Then we obtain the solution in the internal $\left(s<s_{0}-\delta\right)$ and external $\left(s>s_{0}\right)$ homogeneous regions. Finally we match these solutions at the boundaries of the inhomogeneous layer.

\subsection{Solution in the inhomogeneous layer}

Let us introduce the characteristic thickness of the inhomogeneous layer $\ell$. Since the thickness of the inhomogeneous layer varies from $b \delta$ to $a \delta$, it follows that $\ell \ll a$. Let us also introduce the velocity components in the $s$ and $\varphi$-direction, $u$ and $v$. The following formulae are valid in elliptic coordinates (e.g. Korn \& Korn 1961):

$\nabla_{\perp}=\frac{1}{H}\left(\boldsymbol{e}_{s} \frac{\partial}{\partial s}+\boldsymbol{e}_{\varphi} \frac{\partial}{\partial \varphi}\right), \nabla_{\perp} \cdot \boldsymbol{v}=\frac{1}{H^{2}}\left[\frac{\partial(H u)}{\partial s}+\frac{\partial(H v)}{\partial \varphi}\right]$,

where $\boldsymbol{e}_{s}$ and $\boldsymbol{e}_{\varphi}$ are unit vectors in the $s$ and $\varphi$-direction, and the Lamé coefficient $H$ is given by

$H^{2}=\sigma^{2}\left(\sinh ^{2} s+\sin ^{2} \varphi\right)$.

We shall see in what follows that the eignefrequencies of the tube oscillations are of the order of $\omega_{\mathrm{A}}$. Using this fact and (9), we obtain from (8) the estimates

$\frac{1}{H} \frac{\partial P}{\partial s} \sim \rho \omega_{\mathrm{A}} u, \quad \frac{1}{H^{2}} \frac{\partial(H u)}{\partial s} \sim \frac{\omega_{\mathrm{A}}}{\rho V_{\mathrm{A}}^{2}} P$.

Now we notice that $H^{-2} \partial(H u) / \partial s \sim u / \ell$. With this estimate in mind we obtain from (11)

$\frac{1}{H} \frac{\partial P}{\partial s} \sim \frac{\ell P}{L^{2}}$.

Using the approximate formula

$P(s, \varphi)=P\left(s_{0}, \varphi\right)+\frac{\partial P}{\partial s}\left(s-s_{0}\right)$,

and the estimate $\left|s-s_{0}\right| \lesssim \ell / H$, we now obtain, with the account of (12), that $\left|P(s, \varphi)-P\left(s_{0}, \varphi\right)\right| \lesssim(\ell / H)^{2} P$. This result shows that we can neglect the variation of $P$ in the $s$-direction in the inhomogeneous layer and take $P(s, \varphi) \approx P\left(s_{0}, \varphi\right)$. This approximation significantly simplifies the analysis. It was first used by Hollweg (1987) and subsequently by Hollweg \& Yang (1988) to study resonant absorption of MHD waves in a thin inhomogeneous layer in a planar geometry. Recently, it was used by
Ruderman \& Wright (2000) to study nonstationary driven oscillations of a thin planar magnetic cavity, and by Ruderman \& Roberts (2002) to study resonant damping of kink oscillations of a magnetic tube.

The only quantity that we need for what follows is the jump of $u$ across the inhomogeneous layer, $u\left(s_{0}, \varphi\right)-u\left(s_{0}-\delta, \varphi\right)$. Eliminating $v$ from (8) with the use of (9) yields

$$
\frac{\partial(H u)}{\partial s}=\frac{i \omega}{\rho}\left(\frac{H^{2} P}{V_{\mathrm{A}}^{2}}+\frac{1}{\omega^{2}-\omega_{\mathrm{A}}^{2}} \frac{\partial^{2} P}{\partial \varphi^{2}}\right) \text {. }
$$

Since $P$ is determined by its values at the boundaries of the inhomogeneous layer, this equation can be considered as an equation with known right-hand side. In what follows we shall see that the real part of the frequency of a weakly damped eigenmode, $\omega_{\mathrm{r}}$, satisfies the inequality $\omega_{\mathrm{Ai}}<\omega_{\mathrm{r}}<\omega_{\mathrm{Ae}}$. This implies that there is such a value $s_{\mathrm{A}}, s_{0}-\delta<s_{\mathrm{A}}<s_{0}$, that $\omega_{\mathrm{A}}\left(s_{\mathrm{A}}\right)=\omega_{\mathrm{r}}$. For any fixed $\varphi$, the position $s=s_{\mathrm{A}}$ is called the Alfvén resonant position. In the vicinity of this position there are large gradients of the velocity, so that dissipation is important. In weakly dissipative plasmas dissipation is only important in a thin dissipative layer embracing the Alfvén resonant position (e.g., Goosens 1991; Goossens \& Ruderman 1995). In very weakly dissipative plasmas, like the solar coronal plasma, the thickness of the dissipative layer is of order $\ell\left|\omega_{\mathrm{i}} / \omega_{\mathrm{r}}\right|$, where $\omega_{\mathrm{i}}$ is the imaginary part of the eigenfrequency (e.g., Ruderman et al. 1995; Tirry \& Goossens 1996). Inside the dissipative layer there are spatial oscillations of all quantities with the wavelength much smaller than the thickness of the dissipative layer.

In what follows we shall see that $\omega_{\mathrm{i}} / \omega_{\mathrm{r}} \sim \ell / a$, so that the thickness of the dissipative layer is of order $\ell^{2} / a \ll \ell$. This observation inspires us to consider the dissipative layer as a surface of discontinuity and solve the ideal MHD equations to the left and the right of this surface. It turns out that, to connect the solutions to the left and the right of the dissipative layer, it is enough to know the jumps of $u$ and $P$ across the dissipative layer. The formulae expressing these jumps are called the connection formulae.

This method of studying resonant MHD waves in weakly dissipative plasmas was first suggested by Sakurai et al. (1991) and then improved by Goossens et al. (1995) for the so-called driven problem. In the driven problem, plasma oscillations are imposed by an external source of energy, and $\omega$ is assumed to be real. The thickness of the dissipative layer is determined by dissipation. Sakurai et al. (1991) derived the connection formulae for the driven problem. It follows from the results obtained by Ruderman et al. (1995) and Tirry \& Goossens (1996) that the same connection formulae are valid in the case of weakly damped oscillations even in very weakly dissipative plasmas where the thickness of the dissipative layer is determined by the damping rate.

Sakurai et al. (1991) and Goossens et al. (1995) derived the connection formulae in cylindrical geometry under the assumption that all equilibrium quantities depend on the radial coordinate only. However, it is straightforward to generalize these formulae for a particular equilibrium considered in this paper. As a result, we obtain

$\llbracket P \rrbracket=0, \quad \llbracket u \rrbracket=\frac{\pi\left|\omega_{\mathrm{r}}\right|}{H \rho_{\mathrm{A}}|\Delta|} \frac{\partial^{2} P}{\partial \varphi^{2}}$. 
Here $\llbracket f \rrbracket$ is the jump of the function $f(s, \varphi)$ across the dissipative layer, $\rho_{\mathrm{A}}=\rho\left(s_{\mathrm{A}}\right)$, and $\omega_{\mathrm{A}}$ and $H$ are calculated at $s=s_{\mathrm{A}}$. The quantity $\Delta$ is given by

$\Delta=-\left.\frac{\mathrm{d} \omega_{\mathrm{A}}^{2}}{\mathrm{~d} s}\right|_{s=s_{\mathrm{A}}}$.

When we neglected the variation of $P$ across the inhomogeneous layer then, strictly speaking, we only could assume that $P$ is a piece-wise constant function of $s$, in general, discontinuous at $s=s_{\mathrm{A}}$. It follows from the first connection formula in (15) that $P$ is continuous at $s=s_{\mathrm{A}}$ and, therefore, it is independent of $s$ inside the inhomogeneous layer.

Since $\delta \ll s_{0}$, we can neglect the variation of $H$ across the inhomogeneous layer. Then it follows from (14) that

$u=\left\{\begin{aligned} u\left(s_{0}-\delta\right)+\frac{i \omega H\left(s-s_{0}+\delta\right)}{\rho V_{\mathrm{A}}^{2}} P+\frac{i \omega}{H} \frac{\partial^{2} P}{\partial \varphi^{2}} \\ \quad \times \int_{s_{0}-\delta}^{s} \frac{\mathrm{d} s^{\prime}}{\rho\left(s^{\prime}\right)\left[\omega^{2}-\omega_{\mathrm{A}}^{2}\left(s^{\prime}\right)\right]}, \quad s_{0}-\delta<s<s_{\mathrm{A}}, \\ u\left(s_{0}\right)-\frac{i \omega H\left(s_{0}-s\right)}{\rho V_{\mathrm{A}}^{2}} P-\frac{i \omega}{H} \frac{\partial^{2} P}{\partial \varphi^{2}} \\ \quad \times \int_{s}^{s_{0} \frac{\mathrm{d} s^{\prime}}{\rho\left(s^{\prime}\right)\left[\omega^{2}-\omega_{\mathrm{A}}^{2}\left(s^{\prime}\right)\right]},}, \quad s_{\mathrm{A}}<s<s_{0} .\end{aligned}\right.$

Neglecting $\omega_{\mathrm{i}}$ in comparison with $\omega_{\mathrm{r}}$, we obtain from (17)

$$
\begin{aligned}
\llbracket u \rrbracket= & \lim _{\varepsilon \rightarrow+0}\left[u\left(s_{\mathrm{A}}+\varepsilon\right)-u\left(s_{\mathrm{A}}-\varepsilon\right)\right]=u\left(s_{0}\right)-u\left(s_{0}-\delta\right) \\
& -\frac{i \omega_{\mathrm{r}} H \delta}{\rho V_{\mathrm{A}}^{2}} P-\frac{i \omega_{\mathrm{r}}}{H} \frac{\partial^{2} P}{\partial \varphi^{2}} \mathcal{P} \int_{s_{0}-\delta}^{s_{0}} \frac{\mathrm{d} s}{\rho(s)\left[\omega^{2}-\omega_{\mathrm{A}}^{2}(s)\right]},
\end{aligned}
$$

where $\mathcal{P}$ indicates the principal Cauchy part of an integral. Comparing (15) and (18), we obtain

$$
\begin{aligned}
u\left(s_{0}\right)-u\left(s_{0}-\delta\right)= & \frac{\pi\left|\omega_{\mathrm{r}}\right|}{H \rho_{\mathrm{A}}|\Delta|} \frac{\partial^{2} P}{\partial \varphi^{2}}+\frac{i \omega_{\mathrm{r}} H \delta}{\rho V_{\mathrm{A}}^{2}} P \\
& +\frac{i \omega_{\mathrm{r}}}{H} \frac{\partial^{2} P}{\partial \varphi^{2}} \mathcal{P} \int_{s_{0}-\delta}^{s_{0}} \frac{\mathrm{d} s}{\rho(s)\left[\omega^{2}-\omega_{\mathrm{A}}^{2}(s)\right]}
\end{aligned}
$$

\subsection{Solution in the internal region}

Now we obtain the solution in the internal region determined by the inequality $s \leq s_{0}-\delta$. In this region all equilibrium quantities are constant. Eliminating $\boldsymbol{v}$ from (8), we obtain

$\nabla_{\perp}^{2} P+\frac{\omega^{2}-\omega_{\mathrm{Ai}}^{2}}{V_{\mathrm{Ai}}^{2}} P=0$.

The velocity component $u$ is determined by

$u=\frac{-i \omega}{\rho_{\mathrm{i}} H\left(\omega^{2}-\omega_{\mathrm{Ai}}^{2}\right)} \frac{\partial P}{\partial s}$.

In what follows we use the regular perturbation method to find the solution to the problem, and expand $P, u$ and $\omega$ in the series

$$
\begin{aligned}
& P=P_{0}+\epsilon P_{1}+\ldots, \quad u=u_{0}+\epsilon u_{1}+\ldots, \\
& \omega=\omega_{0}+\epsilon \omega_{1}+\ldots,
\end{aligned}
$$

where $\epsilon=\delta / s_{0} \ll 1$. Since $\omega_{\mathrm{i}} / \omega_{\mathrm{r}} \sim \ell / a \sim \epsilon$, we can take $\omega_{0}$ to be real. Using the expression for $\nabla_{\perp}^{2}$ in elliptic coordinates (e.g. Korn \& Korn 1961), we obtain from (20) in the zero order approximation

$\frac{1}{H^{2}}\left(\frac{\partial^{2} P_{0}}{\partial s^{2}}+\frac{\partial^{2} P_{0}}{\partial \varphi^{2}}\right)+\frac{4 \theta_{i}}{\sigma^{2}} P_{0}=0$,

where

$\theta_{\mathrm{i}}=\frac{\sigma^{2}\left(\omega_{0}^{2}-\omega_{\mathrm{Ai}}^{2}\right)}{4 V_{\mathrm{Ai}}^{2}}$

Let us look for the solution to Eq. (23) in the form $P_{0}=$ $S(s) \Phi(\varphi)$. Substitution of this expression in (23) yields

$\frac{S^{\prime \prime}}{S}+4 \theta_{\mathrm{i}} \sinh ^{2} s=-\left(\frac{\Phi^{\prime \prime}}{\Phi}+4 \theta_{\mathrm{i}} \sin ^{2} \varphi\right)$,

where the prime indicates the derivative. Since the left-hand side of this equation depends only on $s$, and the right-hand side only on $\varphi$, they both have to be constant. In particular, it follows that $\Phi$ satisfies the equation

$\Phi^{\prime \prime}+\left(h-2 \theta_{\mathrm{i}} \cos 2 \varphi\right) \Phi=0$,

where $h$ is a constant to be determined. The function $\Phi(\varphi)$ has to be periodic with the period $2 \pi$. Equation (26) is the Mathieu equation. The theory of Mathieu equation states that, for any $\theta_{\mathrm{i}}$, there are two infinite monotonically growing sequences of positive numbers, $\mu_{0}, \mu_{1}, \mu_{2}, \ldots$, and $v_{1}, v_{2}, \ldots$, such that (26) has a periodic solution with the period $2 \pi$ if and only if either $h=\mu_{n}$, or $h=v_{n}$ (e.g., Bateman 1955; Abramowitz \& Stegun 1964). When $h=\mu_{n}$, the corresponding periodic solution is denoted by $\operatorname{ce}_{n}\left(\varphi, \theta_{\mathrm{i}}\right)$. This function is even and has $n$ zeros in the interval $0 \leq \varphi<\pi$. When $h=v_{n}$, the corresponding periodic solution is denoted by $\operatorname{se}_{n}\left(\varphi, \theta_{\mathrm{i}}\right)$. This function is odd and, once again, it has $n$ zeros in the interval $0 \leq \varphi<\pi$.

It is straightforward to see that the points with elliptic coordinates $s=0, \varphi=\varphi_{0}$ and $s=0, \varphi=-\varphi_{0}$ correspond to the same point in the $x y$-plane. This implies that the solution has to satisfy the regularity conditions at $s=0$ :

$P(0, \varphi)=P(0,-\varphi), \quad u(0, \varphi)=-u(0,-\varphi)$.

Using (21), we rewrite the second condition as

$\left.\frac{\partial P(s, \varphi)}{\partial s}\right|_{s=0}=-\left.\frac{\partial P(s,-\varphi)}{\partial s}\right|_{s=0}$.

It follows from (25) and (26) that $S(s)$ has to satisfy the modified Mathieu equation

$S^{\prime \prime}-\left(h-2 \theta_{\mathrm{i}} \cosh 2 s\right) S=0$.

When $h=\mu_{n}$ and $\Phi=\operatorname{ce}_{n}\left(\varphi, \theta_{\mathrm{i}}\right)$, the first equation in (27) is satisfied automatically, while (28) gives $S^{\prime}(0)=0$. With the accuracy up to a multiplicative constant, there is only one solution to (29) satisfying this condition. It is denoted by $\mathrm{Ce}_{n}\left(s, \theta_{\mathrm{i}}\right)$ (Bateman 1955; Abramowitz \& Stegun 1964). When $h=v_{n}$ and $\Phi=\operatorname{se}_{n}\left(\varphi, \theta_{\mathrm{i}}\right),(28)$ is satisfied automatically, while the first equation in (27) gives $S(0)=0$. Once again, with the accuracy up to a multiplicative constant, there is only one solution 
to (29) satisfying this condition. It is denoted by $\operatorname{Se}_{n}\left(s, \theta_{\mathrm{i}}\right)$. Note that $\mathrm{Ce}_{n}\left(s, \theta_{\mathrm{i}}\right)$ is an even and $\operatorname{Se}_{n}\left(s, \theta_{\mathrm{i}}\right)$ an odd function of $s$, and they are related to the functions $\mathrm{ce}_{n}$ and $\mathrm{se}_{n}$ by

$\mathrm{Ce}_{n}(z, \theta)=\mathrm{ce}_{n}(i z, \theta), \quad \operatorname{Se}_{n}(z, \theta)=-i \mathrm{se}_{n}(i z, \theta)$.

The general solution to (23) is given by

$$
\begin{aligned}
P_{0}^{\mathrm{i}}= & C_{0}^{\mathrm{i}} \operatorname{Ce}_{0}\left(s, \theta_{\mathrm{i}}\right) \operatorname{ce}_{0}\left(\varphi, \theta_{\mathrm{i}}\right) \\
& +\sum_{n=1}^{\infty}\left[C_{n}^{\mathrm{i}} \operatorname{Ce}_{n}\left(s, \theta_{\mathrm{i}}\right) \operatorname{ce}_{n}\left(\varphi, \theta_{\mathrm{i}}\right)+D_{n}^{\mathrm{i}} \operatorname{Se}_{n}\left(s, \theta_{\mathrm{i}}\right) \operatorname{se}_{n}\left(\varphi, \theta_{\mathrm{i}}\right)\right]
\end{aligned}
$$

where $C_{n}^{\mathrm{i}}$ and $D_{n}^{\mathrm{i}}$ are arbitrary constants, and we use the superscript " $i$ " to indicate that this is the solution in the internal region.

In the first order approximation we obtain from (20)

$$
\frac{\partial^{2} P_{1}^{\mathrm{i}}}{\partial s^{2}}+\frac{\partial^{2} P_{1}^{\mathrm{i}}}{\partial \varphi^{2}}+\frac{4 H^{2} \theta_{\mathrm{i}}}{\sigma^{2}} P_{1}^{\mathrm{i}}=-\frac{2 \omega_{0} \omega_{1} H^{2}}{V_{\mathrm{Ai}}^{2}} P_{0}^{\mathrm{i}}
$$

\subsection{Solution in the external region}

The motion in the external region is described by (20) and (21), however with $\omega_{\mathrm{Ae}}$ and $V_{\mathrm{Ae}}$ substituted for $\omega_{\mathrm{Ai}}$ and $V_{\mathrm{Ai}}$. We once again use the expansions (22) and arrive at the equation obtained from (23) by substituting $\theta_{\mathrm{e}}$ for $\theta_{\mathrm{i}}$, where

$\theta_{\mathrm{e}}=\frac{\sigma^{2}\left(\omega_{0}^{2}-\omega_{\mathrm{Ae}}^{2}\right)}{4 V_{\mathrm{Ae}}^{2}}$.

Looking for a solution in the form $P_{0}=S(s) \Phi(\varphi)$, we obtain Eqs. (26) and (29) for $\Phi$ and $S$ with $\theta_{\mathrm{e}}$ substituted for $\theta_{\mathrm{i}}$. Similar to the previous subsection, we obtain that either $h=\mu_{n}$ and $\Phi=\operatorname{ce}_{n}\left(\varphi, \theta_{\mathrm{e}}\right), n=0,1,2, \ldots$, or $h=v_{n}$ and $\Phi=\operatorname{se}_{n}\left(\varphi, \theta_{\mathrm{e}}\right)$, $n=1,2, \ldots$.

In what follows we study only non-leaky modes and assume that $\omega_{0}<\omega_{\mathrm{Ae}}$, so that $\theta_{\mathrm{e}}<0$ (Cally 1986). In a non-leaky mode, the perturbation have to vanish far from the tube. This implies the condition

$\lim _{s \rightarrow \infty} S(s)=0$.

With the accuracy up to a multiplicative constant, the solution to the modified Mathieu equation with $\theta_{\mathrm{e}}<0$ satisfying (34) is equal to $\operatorname{Fek}\left(s, \theta_{\mathrm{e}}\right)$ when $h=\mu_{n}$, and it is equal to $\operatorname{Gek}\left(s, \theta_{\mathrm{e}}\right)$ when $h=v_{n}$, where Fek and Gek are the modified Mathieu functions of the third kind (Bateman 1955). Hence, the general solution in the zero order approximation in the external region reads

$$
\begin{aligned}
P_{0}^{\mathrm{e}}= & C_{0}^{\mathrm{e}} \operatorname{Fek}_{0}\left(s, \theta_{\mathrm{e}}\right) \operatorname{ce}_{0}\left(\varphi, \theta_{\mathrm{e}}\right) \\
& +\sum_{n=1}^{\infty}\left[C_{n}^{\mathrm{e}} \operatorname{Fek}_{n}\left(s, \theta_{\mathrm{e}}\right) \operatorname{ce}_{n}\left(\varphi, \theta_{\mathrm{e}}\right)\right. \\
& \left.+D_{n}^{\mathrm{e}} \operatorname{Gek}_{n}\left(s, \theta_{\mathrm{e}}\right) \operatorname{se}_{n}\left(\varphi, \theta_{\mathrm{e}}\right)\right],
\end{aligned}
$$

where $C_{n}^{\mathrm{e}}$ and $D_{n}^{\mathrm{e}}$ are arbitrary constants, and we use the superscript "e" to indicate that this is the solution in the external region.

In the first order approximation we obtain Eq. (32), however with $\theta_{\mathrm{e}}, V_{\mathrm{Ae}}, P_{0}^{\mathrm{e}}$ and $P_{1}^{\mathrm{e}}$ substituted for $\theta_{\mathrm{i}}, V_{\mathrm{Ai}}, P_{0}^{\mathrm{i}}$ and $P_{1}^{\mathrm{i}}$

\subsection{Matching solutions}

To obtain the dispersion equation determining the eigenfrequencies of the tube oscillations, we need to match the solutions in the internal, intermediate and external regions. Since we neglected the pressure variation in the intermediate region, the pressure at the boundary of the internal region has to coincide with the pressure at the boundary of the external region. This condition gives us two equations of the first and second order approximation:

$P_{0}^{\mathrm{e}}=P_{0}^{\mathrm{i}}, \quad P_{1}^{\mathrm{e}}=P_{1}^{\mathrm{i}}-s_{0} \frac{\partial P_{0}^{\mathrm{i}}}{\partial s}$.

To derive the second equation in (36) we have used the expansion $P_{0}^{\mathrm{i}}\left(s_{0}-\delta\right)=P_{0}^{\mathrm{i}}\left(s_{0}\right)-\delta \partial P_{0}^{\mathrm{i}} / \partial s$. All quantities in (36) are calculated at $s=s_{0}$.

The second boundary condition is the continuity of $u$. It has to be satisfied at both boundaries of the intermediate region. This implies that we can substitute $u^{\mathrm{e}}\left(s_{0}\right)$ for $u\left(s_{0}\right)$ and $u^{\mathrm{i}}\left(s_{0}-\delta\right)$ for $u\left(s_{0}-\delta\right)$ in (19). Then, using (21) and a similar equation for the external region, we rewrite (19) as

$$
\begin{gathered}
\left.\frac{i \omega}{\rho_{\mathrm{e}} H\left(\omega_{\mathrm{Ae}}^{2}-\omega^{2}\right)} \frac{\partial P^{\mathrm{e}}}{\partial s}\right|_{s=s_{0}}-\left.\frac{i \omega}{\rho_{\mathrm{i}} H\left(\omega_{\mathrm{Ai}}^{2}-\omega^{2}\right)} \frac{\partial P^{\mathrm{i}}}{\partial s}\right|_{s=s_{0}-\delta} \\
=\frac{\pi\left|\omega_{\mathrm{r}}\right|}{H \rho_{\mathrm{A}}|\Delta|} \frac{\partial^{2} P^{\mathrm{e}}}{\partial \varphi^{2}}+\frac{i \omega_{\mathrm{r}} H \delta}{\rho V_{\mathrm{A}}^{2}} P^{\mathrm{e}} \\
+\frac{i \omega_{\mathrm{r}}}{H} \frac{\partial^{2} P^{\mathrm{e}}}{\partial \varphi^{2}} \mathcal{P} \int_{s_{0}-\delta}^{s_{0}} \frac{\mathrm{d} s}{\rho(s)\left[\omega_{\mathrm{r}}^{2}-\omega_{\mathrm{A}}^{2}(s)\right]}
\end{gathered}
$$

where $P^{\mathrm{e}}$ on the right-hand side of this equation is calculated at $s=s_{0}$. Since $\Delta \sim H \omega_{\mathrm{A}}^{2} / \delta$, we conclude that the right-hand side of (37) is of order $\epsilon$. This, in particular, enables us to substitute $\omega_{0}$ for $\omega_{\mathrm{r}}$ on the right-hand side of (37). Then, making proper expansions, we obtain from (37) in the zero and first order approximation

$$
\begin{gathered}
\frac{1}{\rho_{\mathrm{e}}\left(\omega_{\mathrm{Ae}}^{2}-\omega_{0}^{2}\right)} \frac{\partial P_{0}^{\mathrm{e}}}{\partial s}=\frac{1}{\rho_{\mathrm{i}}\left(\omega_{\mathrm{Ai}}^{2}-\omega_{0}^{2}\right)} \frac{\partial P_{0}^{\mathrm{i}}}{\partial s} \\
\frac{1}{\rho_{\mathrm{e}}\left(\omega_{\mathrm{Ae}}^{2}-\omega_{0}^{2}\right)} \frac{\partial P_{1}^{\mathrm{e}}}{\partial s}-\frac{1}{\rho_{\mathrm{i}}\left(\omega_{\mathrm{Ai}}^{2}-\omega_{0}^{2}\right)} \frac{\partial P_{1}^{\mathrm{i}}}{\partial s} \\
=\frac{\omega_{1}}{\omega_{0}}\left[\frac{\omega_{\mathrm{Ai}}^{2}+\omega_{0}^{2}}{\rho_{\mathrm{i}}\left(\omega_{\mathrm{Ai}}^{2}-\omega_{0}^{2}\right)^{2}} \frac{\partial P_{0}^{\mathrm{i}}}{\partial s}-\frac{\omega_{\mathrm{Ae}}^{2}+\omega_{0}^{2}}{\rho_{\mathrm{e}}\left(\omega_{\mathrm{Ae}}^{2}-\omega_{0}^{2}\right)^{2}} \frac{\partial P_{0}^{\mathrm{e}}}{\partial s}\right] \\
-\frac{s_{0}}{\rho_{\mathrm{i}}\left(\omega_{\mathrm{Ai}}^{2}-\omega_{0}^{2}\right)} \frac{\partial^{2} P_{0}^{\mathrm{i}}}{\partial s^{2}}-\frac{i \pi\left|\omega_{0}\right| s_{0}}{\omega_{0} \rho_{\mathrm{A}}|\Delta| \delta} \frac{\partial^{2} P_{0}^{\mathrm{e}}}{\partial \varphi^{2}}+\frac{H^{2} s_{0}}{\rho V_{\mathrm{A}}^{2}} P_{0}^{\mathrm{e}} \\
+\frac{s_{0}}{\delta} \frac{\partial^{2} P_{0}^{\mathrm{e}}}{\partial \varphi^{2}} \mathcal{P} \int_{s_{0}-\delta}^{s_{0}} \frac{\mathrm{d} s}{\rho(s)\left[\omega_{0}^{2}-\omega_{\mathrm{A}}^{2}(s)\right]}
\end{gathered}
$$

In this equation $P_{0,1}^{i}, P_{0,1}^{\mathrm{e}}$ and all their derivatives are calculated at $s=s_{0}$.

\section{Eigenmodes of a homogeneous tube}

In this section we consider waves in a homogeneous magnetic tube, where there is no inhomogeneous layer, i.e. $\delta=0$. 
In this case the solution is given by the first terms in the expansions (22). Substituting (31) and (35) in the first equation in (36) and in (38), we obtain the system of equations

$$
\begin{aligned}
& C_{0}^{\mathrm{i}} \operatorname{Ce}_{0}\left(s_{0}, \theta_{\mathrm{i}}\right) \mathrm{ce}_{0}\left(\varphi, \theta_{\mathrm{i}}\right)+\sum_{n=1}^{\infty}\left[C_{n}^{\mathrm{i}} \operatorname{Ce}_{n}\left(s_{0}, \theta_{\mathrm{i}}\right) \operatorname{ce}_{n}\left(\varphi, \theta_{\mathrm{i}}\right)\right. \\
& \left.+D_{n}^{\mathrm{i}} \operatorname{Se}_{n}\left(s_{0}, \theta_{\mathrm{i}}\right) \operatorname{se}_{n}\left(\varphi, \theta_{\mathrm{i}}\right)\right]=C_{0}^{\mathrm{e}} \operatorname{Fek}_{0}\left(s_{0}, \theta_{\mathrm{e}}\right) \operatorname{ce}_{0}\left(\varphi, \theta_{\mathrm{e}}\right) \\
& \quad+\sum_{n=1}^{\infty}\left[C_{n}^{\mathrm{e}} \operatorname{Fek}_{n}\left(s_{0}, \theta_{\mathrm{e}}\right) \operatorname{ce}_{n}\left(\varphi, \theta_{\mathrm{e}}\right)\right. \\
& \left.\quad+D_{n}^{\mathrm{e}} \operatorname{Gek}_{n}\left(s_{0}, \theta_{\mathrm{e}}\right) \operatorname{se}_{n}\left(\varphi, \theta_{\mathrm{e}}\right)\right]
\end{aligned}
$$

$$
\begin{aligned}
& \frac{1}{\rho_{\mathrm{i}}\left(\omega_{\mathrm{Ai}}^{2}-\omega_{0}^{2}\right)}\left\{C_{0}^{\mathrm{i}} \operatorname{Ce}_{0}^{\prime}\left(s_{0}, \theta_{\mathrm{i}}\right) \mathrm{ce}_{0}\left(\varphi, \theta_{\mathrm{i}}\right)\right. \\
& \left.\quad+\sum_{n=1}^{\infty}\left[C_{n}^{\mathrm{i}} \operatorname{Ce}_{n}^{\prime}\left(s_{0}, \theta_{\mathrm{i}}\right) \mathrm{ce}_{n}\left(\varphi, \theta_{\mathrm{i}}\right)+D_{n}^{\mathrm{i}} \operatorname{Se}_{n}^{\prime}\left(s_{0}, \theta_{\mathrm{i}}\right) \operatorname{se}_{n}\left(\varphi, \theta_{\mathrm{i}}\right)\right]\right\} \\
& =\frac{1}{\rho_{\mathrm{e}}\left(\omega_{\mathrm{Ae}}^{2}-\omega_{0}^{2}\right)}\left\{C_{0}^{\mathrm{e}} \operatorname{Fek}_{0}^{\prime}\left(s_{0}, \theta_{\mathrm{e}}\right) \mathrm{ce}_{0}\left(\varphi, \theta_{\mathrm{e}}\right)\right. \\
& \quad+\sum_{n=1}^{\infty}\left[C_{n}^{\mathrm{e}} \operatorname{Fek}_{n}^{\prime}\left(s_{0}, \theta_{\mathrm{e}}\right) \mathrm{ce}_{n}\left(\varphi, \theta_{\mathrm{e}}\right)\right. \\
& \left.\left.\quad+D_{n}^{\mathrm{e}} \operatorname{Gek}_{n}^{\prime}\left(s_{0}, \theta_{\mathrm{e}}\right) \operatorname{se}_{n}\left(\varphi, \theta_{\mathrm{e}}\right)\right]\right\}
\end{aligned}
$$

where the prime indicates the derivative with respect to $s$.

The functions $\operatorname{ce}_{n}(\varphi, \theta)$ and $\operatorname{se}_{n}(\varphi, \theta)$ can be expanded in the Fourier series (e.g. Bateman 1955; Abramowitz \& Stegun 1964)

$\operatorname{ce}_{2 m+j}(\varphi, \theta)=\sum_{r=0}^{\infty} A_{2 r+j}^{2 m+j}(\theta) \cos [(2 r+j) \varphi]$,

$\operatorname{se}_{2 m+1+j}(\varphi, \theta)=\sum_{r=0}^{\infty} B_{2 r+1+j}^{2 m+1+j}(\theta) \sin [(2 r+1+j) \varphi]$

where $m=0,1,2, \ldots$ and $j=0,1$. The coefficients $A_{2 r}^{2 m}(\theta)$, $r=0,1,2, \ldots$, are related by recurrence relations, and the same is true for $A_{2 r+1}^{2 m+1}(\theta), B_{2 r+1}^{2 m+1}(\theta)$ and $B_{2 r+2}^{2 m+2}(\theta)$. These recurrence relations can be found in, e.g., Bateman (1955) and Abramowitz \& Stegun (1964). Substituting (42) and (43) in (40) and (41) and collecting terms proportional to $\cos (n \varphi)$ and $\sin (n \varphi)$, we obtain four infinite systems of linear homogeneous algebraic equations with respect to $C_{n}^{\mathrm{i}, \mathrm{e}}$ and $D_{n}^{\mathrm{i}, \mathrm{e}}$ :

$$
\begin{aligned}
& \sum_{m=0}^{\infty} \operatorname{Ce}_{2 m+j}\left(s_{0}, \theta_{\mathrm{i}}\right) A_{2 n+j}^{2 m+j}\left(\theta_{\mathrm{i}}\right) C_{2 m+j}^{\mathrm{i}} \\
& =\sum_{m=0}^{\infty} \operatorname{Fek}_{2 m+j}\left(s_{0}, \theta_{\mathrm{e}}\right) A_{2 n+j}^{2 m+j}\left(\theta_{\mathrm{e}}\right) C_{2 m+j}^{\mathrm{e}}, \\
& \frac{1}{\rho_{\mathrm{i}}\left(\omega_{\mathrm{Ai}}^{2}-\omega_{0}^{2}\right)} \sum_{m=0}^{\infty} \mathrm{Ce}_{2 m+j}^{\prime}\left(s_{0}, \theta_{\mathrm{i}}\right) A_{2 n+j}^{2 m+j}\left(\theta_{\mathrm{i}}\right) C_{2 m+j}^{\mathrm{i}} \\
& =\frac{1}{\rho_{\mathrm{e}}\left(\omega_{\mathrm{Ae}}^{2}-\omega_{0}^{2}\right)} \sum_{m=0}^{\infty} \operatorname{Fek}_{2 m+j}^{\prime}\left(s_{0}, \theta_{\mathrm{e}}\right) A_{2 n+j}^{2 m+j}\left(\theta_{\mathrm{e}}\right) C_{2 m+j}^{\mathrm{e}},
\end{aligned}
$$

and

$$
\begin{aligned}
& \sum_{m=0}^{\infty} S e_{2 m+1+j}\left(s_{0}, \theta_{\mathrm{i}}\right) B_{2 n+1+j}^{2 m+1+j}\left(\theta_{\mathrm{i}}\right) D_{2 m+1+j}^{\mathrm{i}} \\
& \quad=\sum_{m=0}^{\infty} \mathrm{Gek}_{2 m+1+j}\left(s_{0}, \theta_{\mathrm{e}}\right) B_{2 n+1+j}^{2 m+1+j}\left(\theta_{\mathrm{e}}\right) D_{2 m+1+j}^{\mathrm{e}} \\
& \frac{1}{\rho_{\mathrm{i}}\left(\omega_{\mathrm{Ai}}^{2}-\omega_{0}^{2}\right)} \sum_{m=0}^{\infty} S e_{2 m+1+j}^{\prime}\left(s_{0}, \theta_{\mathrm{i}}\right) B_{2 n+1+j}^{2 m+1+j}\left(\theta_{\mathrm{i}}\right) D_{2 m+1+j}^{\mathrm{i}} \\
& =\frac{1}{\rho_{\mathrm{e}}\left(\omega_{\mathrm{Ae}}^{2}-\omega_{0}^{2}\right)} \sum_{m=0}^{\infty} \operatorname{Gek}_{2 m+1+j}^{\prime}\left(s_{0}, \theta_{\mathrm{e}}\right) B_{2 n+1+j}^{2 m+1+j}\left(\theta_{\mathrm{e}}\right) D_{2 m+1+j}^{\mathrm{e}}
\end{aligned}
$$

where $n=0,1,2, \ldots$ Each of the system of Eqs. (44), (45) and (46), (47), represent two systems of equations, one for $j=0$ and the other for $j=1$. The condition of existence of a non-trivial solution to each of these systems is that the corresponding infinite determinant is zero. This conditions gives four dispersion equations determining $\omega_{0}$.

In general, the dispersion equations for $\omega_{0}$ can be solved only numerically. However the analysis is strongly simplified in the approximation of a thin magnetic tube, where $a \ll L$. Since $a=\sigma \cosh s_{0} \geq \sigma$, it follows that $\theta_{\mathrm{e}} \sim \theta_{\mathrm{i}} \sim(a / L)^{2} \ll 1$. Now we use the expansions of $A_{n}^{m}(\theta)$ and $B_{n}^{m}(\theta)$ in power series of $\theta$ (Abramowitz \& Stegun 1964) to obtain

$$
\begin{aligned}
& A_{0}^{0}=2^{-1 / 2}+O\left(\theta^{2}\right), \quad A_{n}^{n}=1+O\left(\theta^{2}\right), \quad B_{n}^{n}=1+O\left(\theta^{2}\right), \\
& A_{n+2 m}^{n}=O\left(\theta^{m}\right), \quad B_{n+2 m}^{n}=O\left(\theta^{m}\right), \quad(m=1,2, \ldots), \\
& A_{n-2 m}^{n}=O\left(\theta^{m}\right), \quad B_{n-2 m}^{n}=O\left(\theta^{m}\right), \quad(m=1,2, \ldots,[n / 2]),
\end{aligned}
$$

where $n=0,1,2, \ldots$, and $[n / 2]$ indicates the integer part of $n / 2$. Using the relations (30), the Fourier expansions (42) and (43), and the asymptotic formulae (48), we obtain the asymptotics valid for $|\theta|^{1 / 2} \mathrm{e}^{s} \ll 1$ :

$\mathrm{Ce}_{0}(s, \theta) \sim 2^{-1 / 2}, \quad \mathrm{Ce}_{n}(s, \theta) \sim \cosh (n s)$,

$\operatorname{Se}_{n}(s, \theta) \sim \sinh (n s)$,

where $n=1,2, \ldots$

Let us introduce new variables

$$
\begin{aligned}
& \widetilde{C}_{0}^{\mathrm{e}}=\frac{p_{0}}{\pi} C_{0}^{\mathrm{e}}, \quad \widetilde{C}_{2 m-1}^{\mathrm{e}}=\frac{(-1)^{m} p_{2 m-1}}{\pi\left|\theta_{\mathrm{e}}\right|^{1 / 2} A_{1}^{2 m-1}} C_{2 m-1}^{\mathrm{e}}, \\
& \widetilde{C}_{2 m}^{\mathrm{e}}=\frac{(-1)^{m} p_{2 m}}{2 \pi m A_{0}^{2 m}} C_{2 m}^{\mathrm{e}}, \\
& \widetilde{D}_{2 m-1}^{\mathrm{e}}=\frac{(-1)^{m} q_{2 m-1}}{\pi\left|\theta_{\mathrm{e}}\right|^{1 / 2} B_{1}^{2 m-1}} D_{2 m-1}^{\mathrm{e}}, \quad \widetilde{D}_{2 m}^{\mathrm{e}}=\frac{2 m(-1)^{m} q_{2 m}}{\pi \theta_{\mathrm{e}} B_{2}^{2 m}} D_{2 m}^{\mathrm{e}},
\end{aligned}
$$

where $m=1,2, \ldots$ We substitute (50) in the four systems of equations, the first and second given by (44), (45) with $j=0,1$, and the third and fourth given by (46), (47) with $j=0,1$. As a result, we obtain four infinite systems of linear homogeneous algebraic equations, the first one for $C_{2 m}^{\mathrm{i}}$ and $\widetilde{C}_{2 m}^{\mathrm{e}}$, the second one for $C_{2 m+1}^{\mathrm{i}}$ and $\widetilde{C}_{2 m+1}^{\mathrm{e}}$, the third one for $D_{2 m+1}^{\mathrm{i}}$ and $\widetilde{D}_{2 m+1}^{\mathrm{e}}$, and the fourth one for $D_{2 m+2}^{\mathrm{i}}$ and $\widetilde{D}_{2 m+2}^{\mathrm{e}}$, where $m=0,1,2, \ldots$ Using (48), (49), and (A.11)-(A.15), it is straightforward to 
show that all elements of the infinite matrices of the four systems except the diagonal elements tend to zero as $\theta_{\mathrm{i}, \mathrm{e}} \rightarrow 0$. Then, in the leading order approximation with respect to $a / L$, the first and second systems reduce to

$$
\left.\begin{array}{l}
2^{1 / 2} C_{0}^{\mathrm{i}}=-\left(\ln \left|\theta_{\mathrm{e}}\right|+2 s_{0}+2 \gamma-2 \ln 2\right) \widetilde{C}_{0}^{\mathrm{e}}, \\
\rho_{\mathrm{e}}^{-1}\left(\omega_{\mathrm{Ae}}^{2}-\omega_{0}^{2}\right)^{-1} \widetilde{C}_{0}^{\mathrm{e}}=0, \\
\cosh \left(n s_{0}\right) C_{n}^{\mathrm{i}}=\mathrm{e}^{-n s_{0}} \widetilde{C}_{n}^{\mathrm{e}}, \\
\frac{\sinh \left(n s_{0}\right)}{\rho_{\mathrm{i}}\left(\omega_{\mathrm{Ai}}^{2}-\omega_{0}^{2}\right)} C_{n}^{\mathrm{i}}=-\frac{\mathrm{e}^{-n s_{0}}}{\rho_{\mathrm{e}}\left(\omega_{\mathrm{Ae}}^{2}-\omega_{0}^{2}\right)} \widetilde{C}_{n}^{\mathrm{e}},
\end{array}\right\}
$$

while the third and fourth system reduce to

$$
\left.\begin{array}{l}
\sinh \left(n s_{0}\right) D_{n}^{\mathrm{i}}=\mathrm{e}^{-n s_{0}} \widetilde{D}_{n}^{\mathrm{e}}, \\
\frac{\cosh \left(n s_{0}\right)}{\rho_{\mathrm{i}}\left(\omega_{\mathrm{Ai}}^{2}-\omega_{0}^{2}\right)} D_{n}^{\mathrm{i}}=-\frac{\mathrm{e}^{-n s_{0}}}{\rho_{\mathrm{e}}\left(\omega_{\mathrm{Ae}}^{2}-\omega_{0}^{2}\right)} \widetilde{D}_{n}^{\mathrm{e}},
\end{array}\right\}
$$

where $n=1,2, \ldots$

It is obvious that system (51) has only the trivial solution $C_{0}^{\mathrm{e}}=\widetilde{C}_{0}^{\mathrm{e}}=0$ for any value of $\omega_{0}$. A non-trivial solution of this system would give us the only wave mode that changes the area of the tube. It would be similar to the axisymmetric wave propagating along a thin magnetic tube with a circular crosssection. This wave propagates with the tube speed $c_{\mathrm{T}}$ (Defouw 1976), which is zero in a cold plasma. This implies that the axisymmetric wave cannot be described in the cold plasma approximation. The non-existence of a non-trivial solution to system (51) is in a good agreement with this result.

A non-trivial solution to system (52) exists only when the determinant of this system is zero. This condition results in

$\omega_{0}^{2}=\omega_{n c}^{2} \equiv \frac{\rho \omega_{\mathrm{A}}^{2}\left[1+\tanh \left(n s_{0}\right)\right]}{\rho_{\mathrm{i}}+\rho_{\mathrm{e}} \tanh \left(n s_{0}\right)}$.

Similarly, the condition of existence of a non-trivial solution to system (53) results in

$\omega_{0}^{2}=\omega_{n s}^{2} \equiv \frac{\rho \omega_{\mathrm{A}}^{2}\left[1+\tanh \left(n s_{0}\right)\right]}{\rho_{\mathrm{i}} \tanh \left(n s_{0}\right)+\rho_{\mathrm{e}}}$.

When deriving Eqs. (54) and (55), we have taken into account that $\rho_{\mathrm{e}} \omega_{\mathrm{Ae}}^{2}=\rho_{\mathrm{i}} \omega_{\mathrm{Ai}}^{2}=\rho \omega_{\mathrm{A}}^{2}$. The tube boundary perturbed by the modes corresponding to $\omega_{n c}$ and $\omega_{n s}$ is shown in Fig. 3 for $n=1,2,3$. It is straightforward to show that

$$
\begin{aligned}
& \omega_{1 c}<\omega_{2 c}<\omega_{3 c}<\ldots, \\
& \omega_{1 s}>\omega_{2 s}>\omega_{3 s}>\ldots
\end{aligned}
$$

In addition,

$\lim _{n \rightarrow \infty} \omega_{n c}^{2}=\lim _{n \rightarrow \infty} \omega_{n s}^{2}=\omega_{k}^{2} \equiv \frac{2 \rho \omega_{\mathrm{A}}^{2}}{\rho_{\mathrm{i}}+\rho_{\mathrm{e}}}$,

where $\omega_{k}$ is the frequency of the kink mode of a thin magnetic tube with a circular cross-section (e.g. Ryutov \& Ryutova 1976; Edwin \& Roberts 1983). It follows from (56) and (57) that $\omega_{n c}<\omega_{m s}$ for any $n \geq 1$ and $m \geq 1$

The limit $s_{0} \rightarrow \infty$ corresponds to a tube with a circular cross-section, because in this limit $a / b \rightarrow 1$. In this limit we
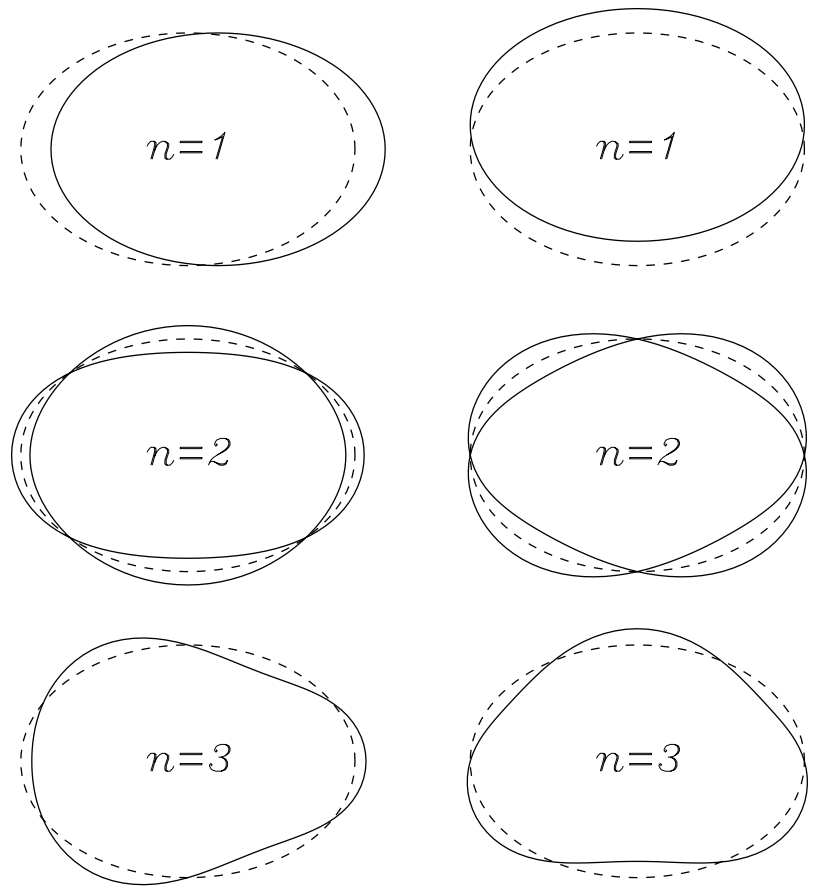

Fig. 3. A sketch of the tube boundary perturbed by eigenmodes. The upper, middle and bottom panels correspond to $n=1, n=2$ and $n=3$. The left panels correspond to $\omega_{n c}$, and the right to $\omega_{n s}$. The dashed and solid lines show the unperturbed and perturbed boundaries respectively. In the middle panel the perturbed boundary is shown for two successive moments of time separated by a half period.

obtain a well-know result for waves in thin magnetic tubes with circular cross-sections (e.g. Spruit 1982):

$\omega_{n c}=\omega_{n s}=\omega_{k} \quad(n=1,2, \ldots)$.

In the opposite limit, where $s_{0} \rightarrow 0$, the tube cross-section is shrunk into the interval $[-\sigma, \sigma]$ of the $x$-axis. In this limit

$\omega_{n c}=\omega_{\mathrm{Ai}}, \quad \omega_{n s}=\omega_{\mathrm{Ae}} \quad(n=1,2, \ldots)$.

These results are not surprising at all. In the modes corresponding to $\omega_{n c}$, the motion is along the $x$-axis, so it does not perturbed the external plasma. As a result, the magnetic field lines inside the tube oscillate with their own Alfvén frequency, which is equal to $\omega_{\mathrm{Ai}}$. In the modes corresponding to $\omega_{n s}$, the motion is perpendicular to the $x$-axis, and it perturbes a region of the external plasma with the characteristic size $\sigma$. The mass of the plasma inside the tube can be neglected in comparison with the mass of the plasma in this region. As a result, the plasma oscillates with the frequency equal to the external Alfvén frequency $\omega_{\mathrm{Ae}}$. For $n=1$ this result recovers the corresponding result for kink oscillations of a magnetic slab (e.g. Enwin \& Roberts 1982).

Expressions (54) and (55) take especially simple forms for $n=1$ :

$\omega_{1 c}^{2}=\frac{\rho \omega_{\mathrm{A}}^{2}(a+b)}{a \rho_{\mathrm{i}}+b \rho_{\mathrm{e}}}, \quad \omega_{1 s}^{2}=\frac{\rho \omega_{\mathrm{A}}^{2}(a+b)}{b \rho_{\mathrm{i}}+a \rho_{\mathrm{e}}}$.

Recall that $a$ and $b$ are the small and large half-axis of the elliptic cross-section. When $a=b, \omega_{1 c}=\omega_{1 s}=\omega_{k}$. 
Let us study polarization of the two kink modes. When $\omega_{0}=\omega_{1 c}$, it follows from (51)-(53) that

$C_{n}^{\mathrm{i}}=\widetilde{C}_{n}^{\mathrm{e}}=0 \quad(n=0,2,3, \ldots), \quad \widetilde{C}_{1}^{\mathrm{e}}=\frac{1}{2}\left(\mathrm{e}^{2 s_{0}}+1\right) C_{1}^{\mathrm{i}}$,

$D_{n}^{\mathrm{i}}=\widetilde{D}_{n}^{\mathrm{e}}=0 \quad(n=1,2, \ldots)$.

Similarly,

$C_{n}^{\mathrm{i}}=\widetilde{C}_{n}^{\mathrm{e}}=0 \quad(n=0,1,2, \ldots)$,

$D_{n}^{\mathrm{i}}=\widetilde{D}_{n}^{\mathrm{e}}=0 \quad(n=2,3, \ldots) \quad \widetilde{D}_{1}^{\mathrm{e}}=\frac{1}{2}\left(\mathrm{e}^{2 s_{0}}-1\right) D_{1}^{\mathrm{i}}$,

when $\omega_{0}=\omega_{1 s}$. Using (42), (43) and (48) we obtain that, for $\left|\theta_{\mathrm{i}, \mathrm{e}}\right| \ll 1$,

$\operatorname{ce}_{1}\left(\varphi, \theta_{\mathrm{i}, \mathrm{e}}\right) \approx \cos \varphi, \quad \operatorname{se}_{1}\left(\varphi, \theta_{\mathrm{i}, \mathrm{e}}\right) \approx \sin \varphi$.

It follows from (50), (61), (62), (A.12) and (A.14) that, for $\left|\theta_{\mathrm{e}}\right| \ll 1$,

$C_{1}^{\mathrm{e}} \operatorname{Fek}_{1}\left(s, \theta_{\mathrm{e}}\right) \approx \frac{1}{2}\left(\mathrm{e}^{2 s_{0}}+1\right) C_{1}^{\mathrm{i}} \mathrm{e}^{-s}$,

$D_{1}^{\mathrm{e}} \operatorname{Gek}_{1}\left(s, \theta_{\mathrm{e}}\right) \approx \frac{1}{2}\left(\mathrm{e}^{2 s_{0}}-1\right) D_{1}^{\mathrm{i}} \mathrm{e}^{-s}$.

Then, making use of (49) and (61)-(64), we obtain from (31) and (35)

$P_{0}^{\mathrm{i}}=C_{1}^{\mathrm{i}} \cosh s \cos \varphi, \quad P_{0}^{\mathrm{e}}=\frac{1}{2}\left(\mathrm{e}^{2 s_{0}}+1\right) C_{1}^{\mathrm{i}} \mathrm{e}^{-s} \cos \varphi$,

when $\omega_{0}=\omega_{1 c}$, and

$P_{0}^{\mathrm{i}}=D_{1}^{\mathrm{i}} \sinh s \sin \varphi, \quad P_{0}^{\mathrm{e}}=\frac{1}{2}\left(\mathrm{e}^{2 s_{0}}-1\right) D_{1}^{\mathrm{i}} \mathrm{e}^{-s} \sin \varphi$,

when $\omega_{0}=\omega_{1 s}$. Using Eqs. (8), (65), (66), and the relations between unit vectors of elliptic, $\boldsymbol{e}_{s}$ and $\boldsymbol{e}_{\varphi}$, and Cartesian coordinates, $\boldsymbol{e}_{x}$ and $\boldsymbol{e}_{y}$,

$\boldsymbol{e}_{s}=H^{-1}\left(\boldsymbol{e}_{x} \sinh s \cos \varphi+\boldsymbol{e}_{y} \cosh s \sin \varphi\right)$,

$\boldsymbol{e}_{\varphi}=H^{-1}\left(-\boldsymbol{e}_{x} \cosh s \sin \varphi+\boldsymbol{e}_{y} \sinh s \cos \varphi\right)$,

we obtain for the $x$ and $y$-component of the velocity, $v_{x}$ and $v_{y}$,

$v_{x}=v_{0 c}, \quad v_{y}=0 \quad\left(\omega_{0}=\omega_{1 c}\right)$,

$v_{x}=0, \quad v_{y}=v_{0 s} \quad\left(\omega_{0}=\omega_{1 s}\right)$.

Here $v_{0 c}$ is a constant proportional to $C_{1}^{\mathrm{i}}$, and $v_{0 s}$ is a constant proportional to $D_{1}^{\mathrm{i}}$. Hence, the kink oscillation with the frequency $\omega_{1 c}$ is polarized in the direction of the large axis of the elliptic cross-section. The kink oscillation with the frequency $\omega_{1 s}$ is polarized in the direction of the small axis of the elliptic cross-section.

Let us study the dependences of $\omega_{1 c}$ and $\omega_{1 s}$ on $a / b$. Using (57) and (60) we obtain

$\frac{\omega_{1 c}^{2}}{\omega_{k}^{2}}=\frac{(1+a / b)\left(1+\rho_{\mathrm{i}} / \rho_{\mathrm{e}}\right)}{2\left[1+(a / b)\left(\rho_{\mathrm{i}} / \rho_{\mathrm{e}}\right)\right]}, \frac{\omega_{1 s}^{2}}{\omega_{k}^{2}}=\frac{(1+a / b)\left(1+\rho_{\mathrm{i}} / \rho_{\mathrm{e}}\right)}{2\left(a / b+\rho_{\mathrm{i}} / \rho_{\mathrm{e}}\right)}$.

In Fig. 4 the dependences of $\omega_{1 c} / \omega_{k}$ and $\omega_{1 s} / \omega_{k}$ on $a / b$ are shown for $\rho_{\mathrm{i}} / \rho_{\mathrm{e}}=10$, which is typical value for coronal loops. When $a / b \rightarrow \infty, \omega_{1 c} / \omega_{k} \rightarrow \sqrt{\left(\rho_{\mathrm{i}}+\rho_{\mathrm{e}}\right) /\left(2 \rho_{\mathrm{i}}\right)} \approx 0.742$ and $\omega_{1 s} / \omega_{k} \rightarrow \sqrt{\left(\rho_{\mathrm{i}}+\rho_{\mathrm{e}}\right) /\left(2 \rho_{\mathrm{e}}\right)} \approx 2.345$. The limiting value for $\omega_{1 c} / \omega_{k}$ is shown in Fig. 4 by the dotted horizontal line. The quantity $\omega_{1 s} / \omega_{k}$ becomes close to its limiting value only for unrealistically large values of $a / b$, so that it is not shown in Fig. 4.

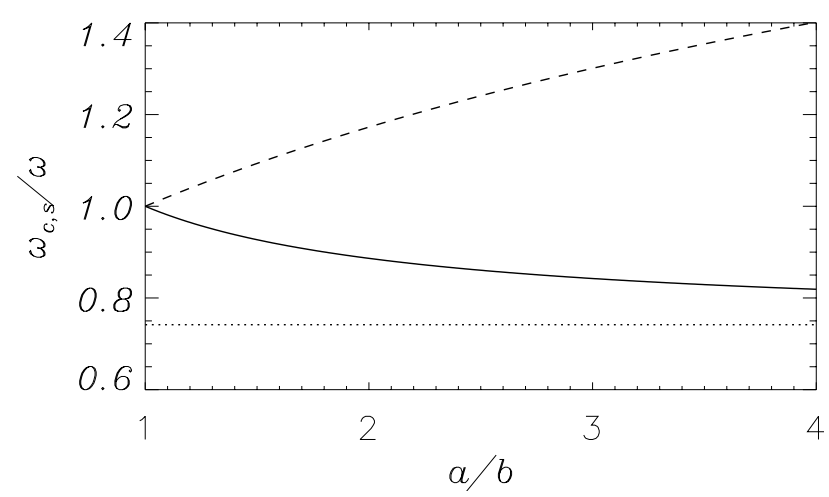

Fig. 4. The solid and dashed curves show the dependences of $\omega_{1 c} / \omega_{k}$ and $\omega_{1 s} / \omega_{k}$ on $a / b$ respectively for $\rho_{\mathrm{i}} / \rho_{\mathrm{e}}=10$. The dotted horizontal line shows the limiting value of $\omega_{1 c} / \omega_{k}$ for $a / b \rightarrow \infty$.

\section{Resonant damping of kink oscillations}

In this section we study resonant damping of the two linearly polarized kink modes in the long wavelength approximation. We start our calculation with determining $P_{1}^{\mathrm{i}}$ and $P_{1}^{\mathrm{e}}$. To do this we use (32) and a similar equation for the external region with $a / L \ll 1$. Then, neglecting terms of order $(a / L)^{2}$, we conclude that $P_{1}^{\mathrm{i}}$ and $P_{1}^{\mathrm{e}}$ satisfy

$\frac{\partial^{2} P_{1}^{\mathrm{i}, \mathrm{e}}}{\partial s^{2}}+\frac{\partial^{2} P_{1}^{\mathrm{i}, \mathrm{e}}}{\partial \varphi^{2}}=0$.

Taking into account that $P_{1}^{\mathrm{i}}$ has to satisfy the first boundary condition in (27) and the boundary condition (28), we arrive at

$P_{1}^{\mathrm{i}}=U_{0}^{\mathrm{i}}+\sum_{n=1}^{\infty}\left[U_{n}^{\mathrm{i}} \cosh (n s) \cos (n \varphi)+W_{n}^{\mathrm{i}} \sinh (n s) \sin (n \varphi)\right]$,

where $U_{n}^{\mathrm{i}}$ and $W_{n}^{\mathrm{i}}$ are arbitrary constants. $P_{1}^{\mathrm{e}}$ has to vanish as $s \rightarrow \infty$. The solution to (71) satisfying this condition is

$P_{1}^{\mathrm{e}}=\sum_{n=1}^{\infty} \mathrm{e}^{-n s}\left[U_{n}^{\mathrm{e}} \cos (n \varphi)+W_{n}^{\mathrm{e}} \sin (n \varphi)\right]$,

where once again $U_{n}^{\mathrm{e}}$ and $W_{n}^{\mathrm{e}}$ are arbitrary constants. Now we substitute (65), (66), (72) and (73) in the second equation in (36) and in (39), neglect terms that tend to zero as $a / L \rightarrow 0$, and collect the terms proportional either to $\cos \varphi$, or to $\sin \varphi$. As a result we arrive at equations

$U_{1}^{\mathrm{i}} \cosh s_{0}-U_{1}^{\mathrm{e}} \mathrm{e}^{-s_{0}}=C_{1}^{\mathrm{i}} s_{0} \sinh s_{0}$,

$$
\begin{aligned}
& \frac{\sinh s_{0}}{\rho_{\mathrm{i}}\left(\omega_{\mathrm{Ai}}^{2}-\omega_{1 c}^{2}\right)} U_{1}^{\mathrm{i}}+\frac{\mathrm{e}^{-s_{0}}}{\rho_{\mathrm{e}}\left(\omega_{\mathrm{Ae}}^{2}-\omega_{1 c}^{2}\right)} U_{1}^{\mathrm{e}} \\
& =-C_{1}^{\mathrm{i}}\left\{\frac{i \pi s_{0} \cosh s_{0}}{\rho_{\mathrm{A}}|\Delta| \delta}-\frac{s_{0} \cosh s_{0}}{\rho_{\mathrm{i}}\left(\omega_{\mathrm{Ai}}^{2}-\omega_{1 c}^{2}\right)}\right. \\
& +\frac{\omega_{1}}{\omega_{1 c}}\left[\frac{\left(\omega_{\mathrm{Ai}}^{2}+\omega_{1 c}^{2}\right) \sinh s_{0}}{\rho_{\mathrm{i}}\left(\omega_{\mathrm{Ai}}^{2}-\omega_{1 c}^{2}\right)^{2}}+\frac{\left(\omega_{\mathrm{Ae}}^{2}+\omega_{1 c}^{2}\right) \cosh s_{0}}{\rho_{\mathrm{e}}\left(\omega_{\mathrm{Ae}}^{2}-\omega_{1 c}^{2}\right)^{2}}\right] \\
& \left.-\frac{s_{0} \cosh s_{0}}{\delta} \mathcal{P} \int_{s_{0}-\delta}^{s_{0}} \frac{\mathrm{d} s}{\rho(s)\left[\omega_{1 c}^{2}-\omega_{\mathrm{A}}^{2}(s)\right]}\right\},
\end{aligned}
$$


when $\omega_{0}=\omega_{1 c}$. For $\omega_{0}=\omega_{1 s}$ we obtain

$W_{1}^{\mathrm{i}} \sinh s_{0}-W_{1}^{\mathrm{e}} \mathrm{e}^{-s_{0}}=D_{1}^{\mathrm{i}} s_{0} \cosh s_{0}$,

$$
\begin{aligned}
& \frac{\cosh s_{0}}{\rho_{\mathrm{i}}\left(\omega_{\mathrm{Ai}}^{2}-\omega_{1 c}^{2}\right)} W_{1}^{\mathrm{i}}+\frac{\mathrm{e}^{-s_{0}}}{\rho_{\mathrm{e}}\left(\omega_{\mathrm{Ae}}^{2}-\omega_{1 c}^{2}\right)} W_{1}^{\mathrm{e}} \\
& =-D_{1}^{\mathrm{i}}\left\{\frac{i \pi s_{0} \sinh s_{0}}{\rho_{\mathrm{A}}|\Delta| \delta}-\frac{s_{0} \sinh s_{0}}{\rho_{\mathrm{i}}\left(\omega_{\mathrm{Ai}}^{2}-\omega_{1 s}^{2}\right)}\right. \\
& +\frac{\omega_{1}}{\omega_{1 s}}\left[\frac{\left(\omega_{\mathrm{Ai}}^{2}+\omega_{1 s}^{2}\right) \cosh s_{0}}{\rho_{\mathrm{i}}\left(\omega_{\mathrm{Ai}}^{2}-\omega_{1 s}^{2}\right)^{2}}+\frac{\left(\omega_{\mathrm{Ae}}^{2}+\omega_{1 s}^{2}\right) \sinh s_{0}}{\rho_{\mathrm{e}}\left(\omega_{\mathrm{Ae}}^{2}-\omega_{1 s}^{2}\right)^{2}}\right] \\
& \left.-\frac{s_{0} \sinh s_{0}}{\delta} \mathcal{P} \int_{s_{0}-\delta}^{s_{0}} \frac{\mathrm{d} s}{\rho(s)\left[\omega_{1 s}^{2}-\omega_{\mathrm{A}}^{2}(s)\right]}\right\} .
\end{aligned}
$$

The system of Eqs. (74) and (75) is an inhomogeneous linear system of algebraic equations for $U_{1}^{\mathrm{i}}$ and $U_{1}^{\mathrm{e}}$. Its determinant coincides with the determinant of the system (52) with $n=1$. Since the latter is zero when $\omega_{0}=\omega_{1 c}$, system (74), (75) has a solution only if its right-hand side satisfies the compatibility condition. This condition determines $\omega_{1}$ for the mode with $\omega_{0}=\omega_{1 c}$. Similarly, the determinant of the system of Eqs. (76) and (77) is also zero, and the corresponding compatibility condition determines $\omega_{1}$ for the mode with $\omega_{0}=\omega_{1 s}$.

The real part of $\omega_{1}$ gives only a small correction to $\omega_{0}$ and can be neglected. In contrast, the imaginary part of $\omega_{1}$ is very important because it describes resonant damping of the oscillations. Introducing the decrement $\gamma=-\epsilon \mathfrak{J}\left(\omega_{1}\right)$, where $\mathfrak{J}$ indicates the imaginary part of a quantity, we obtain

$\gamma_{c}=\frac{\pi a^{2} b \omega_{1 c}^{3}\left(\rho_{\mathrm{i}}-\rho_{\mathrm{e}}\right)^{2}}{2|\Delta|(a+b)\left(a \rho_{\mathrm{i}}+b \rho_{\mathrm{e}}\right)^{2}}$,

$\gamma_{s}=\frac{\pi a b^{2} \omega_{1 s}^{3}\left(\rho_{\mathrm{i}}-\rho_{\mathrm{e}}\right)^{2}}{2|\Delta|(a+b)\left(b \rho_{\mathrm{i}}+a \rho_{\mathrm{e}}\right)^{2}}$.

It is straightforward to show that, for not very large eccentricity $\varepsilon, \gamma_{c} \sim \gamma_{s} \sim \delta \omega_{1 c, s}$, where $a \delta$ is the maximum and $b \delta$ the minimum thickness of the inhomogeneous layer at the tube boundary. When $a=b, \gamma_{c}$ and $\gamma_{s}$ coincide with the decrement of the kink mode oscillation of a thin tube with circular cross-section given by Eq. (56) of Ruderman \& Roberts (2002).

Let us study how $\gamma_{c}$ and $\gamma_{s}$ depend on $a / b$. We assume that the (small) ratio of the area of the inhomogeneous region to the area of the total tube cross-section remains constant when $a / b$ varies. Since the equations of the boundaries of the inhomogeneous region are $s=s_{0}-\delta$ and $s=s_{0}$, and $\delta \ll 1$, the thickness of this region is approximately equal to $H\left(s_{0}, \varphi\right) \delta$. The length of an infinitesimal arc of the boundary corresponding to the variation of $\varphi$ from $\varphi$ to $\varphi+\mathrm{d} \varphi$ is $H \mathrm{~d} \varphi$. Hence, the area of the inhomogeneous region, $S_{\text {in }}$, is given by

$$
\begin{aligned}
S_{\text {in }} & =\delta \int_{0}^{2 \pi} H^{2}\left(s_{0}, \varphi\right) \mathrm{d} \varphi=\delta \sigma^{2} \int_{0}^{2 \pi}\left(\sinh ^{2} s_{0}+\sin ^{2} \varphi\right) \mathrm{d} \varphi \\
& =\pi \delta \sigma^{2}\left(1+2 \sinh ^{2} s_{0}\right)=\pi \delta\left(a^{2}+b^{2}\right) .
\end{aligned}
$$

Since the area of the cross-section is equal to $S_{\mathrm{cr}}=\pi a b$, the condition that $S_{\text {in }} / S_{\text {cr }}=$ const. reduces to

$\delta=\delta_{0} \frac{2 a / b}{1+(a / b)^{2}}$,

where $\delta_{0}$ corresponds to the circular cross-section $(a=b)$.

In what follows we assume that the density varies linearly in the inhomogeneous region in the direction perpendicular to the region boundary. Since $\delta \ll s_{0}$, it is equivalent to the assumption that $\rho$ is a linear function of $s$, i.e. $\rho$ in the inhomogeneous region is given by

$\rho=\rho_{\mathrm{e}}+\left(s_{0}-s\right)\left(\rho_{\mathrm{i}}-\rho_{\mathrm{e}}\right) / \delta$.

The conditions $\omega_{1 c}^{2}=\omega_{\mathrm{A}}^{2}$ and $\omega_{1 s}^{2}=\omega_{\mathrm{A}}^{2}$ reduce to $\rho=\rho_{\mathrm{A} c}$ and $\rho=\rho_{\mathrm{A} s}$ respectively, where

$\rho_{\mathrm{A} c}=\frac{a \rho_{\mathrm{i}}+b \rho_{\mathrm{e}}}{a+b}, \quad \rho_{\mathrm{A} s}=\frac{a \rho_{\mathrm{e}}+b \rho_{\mathrm{i}}}{a+b}$.

Using (16) and (81) we immediately obtain

$\Delta=-\frac{\pi^{2} \rho V_{\mathrm{A}}^{2}\left(\rho_{\mathrm{i}}-\rho_{\mathrm{e}}\right)}{\rho_{\mathrm{A}}^{2} L^{2} \delta}$

Then, with the aid of (82), we arrive at

$\Delta_{c}=-\frac{\pi^{2} \rho V_{\mathrm{A}}^{2}(a+b)^{2}\left(\rho_{\mathrm{i}}-\rho_{\mathrm{e}}\right)}{L^{2} \delta\left(a \rho_{\mathrm{i}}+b \rho_{\mathrm{e}}\right)^{2}}$
$\Delta_{s}=-\frac{\pi^{2} \rho V_{\mathrm{A}}^{2}(a+b)^{2}\left(\rho_{\mathrm{i}}-\rho_{\mathrm{e}}\right)}{L^{2} \delta\left(a \rho_{\mathrm{e}}+b \rho_{\mathrm{i}}\right)^{2}}$

Now, using $\gamma$ to indicate the common value of $\gamma_{c}$ and $\gamma_{s}$ for $a=b$, we obtain

$\frac{\gamma_{c}}{\gamma}=\frac{2^{5 / 2}(a / b)^{3}\left(1+\rho_{\mathrm{i}} / \rho_{\mathrm{e}}\right)^{3 / 2}}{\left[1+(a / b)^{2}\right](1+a / b)^{3 / 2}\left[1+(a / b)\left(\rho_{\mathrm{i}} / \rho_{\mathrm{e}}\right)\right]^{3 / 2}}$,

$\frac{\gamma_{s}}{\gamma}=\frac{2^{5 / 2}(a / b)^{2}\left(1+\rho_{\mathrm{i}} / \rho_{\mathrm{e}}\right)^{3 / 2}}{\left[1+(a / b)^{2}\right](1+a / b)^{3 / 2}\left(a / b+\rho_{\mathrm{i}} / \rho_{\mathrm{e}}\right)^{3 / 2}}$.

It is easy to see that $\gamma_{c} / \gamma \rightarrow 0$ and $\gamma_{s} / \gamma \rightarrow 0$ when $a / b \rightarrow \infty$. It can be shown that $\gamma_{c}<\gamma$ for any $a / b>1$. In Fig. 5 the dependences of $\gamma_{c} / \gamma$ and $\gamma_{s} / \gamma$ on $a / b$ are shown for $\rho_{\mathrm{i}}=10 \rho_{\mathrm{e}}$. We can see that, for moderate values of $a / b(a / b \lesssim 2) \gamma_{c}$ and $\gamma_{s}$ do not differ from $\gamma$ very much.

\section{Summary and discussion}

In this paper we have studied the damped oscillations of a thin straight magnetic tube with an elliptic cross-section in a cold ideal plasma. The damping of the oscillations is due to resonant absorption in a thin inhomogeneous layer at the tube boundary. Our main results are:

(i) There are two infinite sequences of the tube eigenmodes, $\left\{\omega_{n c}\right\}$ and $\left\{\omega_{n s}\right\}(n=1,2, \ldots)$, corresponding to the global modes of the tube. The sequence $\left\{\omega_{n c}\right\}$ is monotonically increasing, and the sequence $\left\{\omega_{n s}\right\}$ is monotonically decreasing. When $n \rightarrow \infty, \omega_{n c, s} \rightarrow \omega_{k}$, where $\omega_{k}$ is the frequency of kink oscillations of a tube with a circular cross-section. The modes corresponding to $\omega_{n c, s}$ have $2 n$ nodes at the tube boundary. 


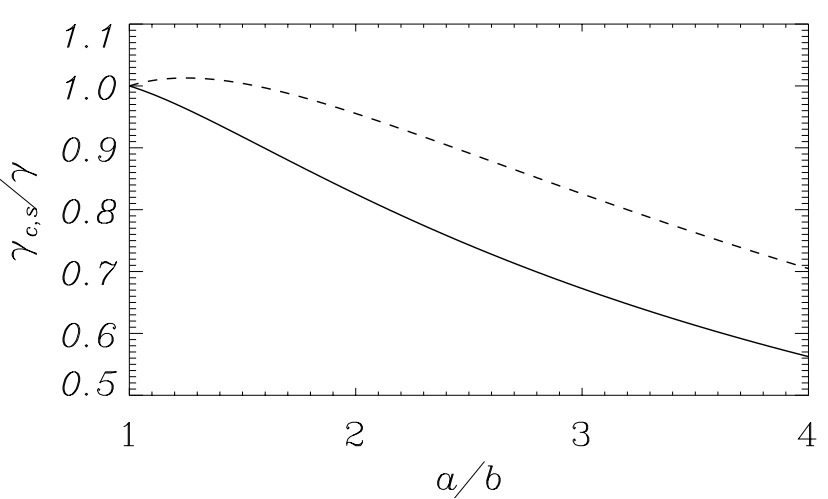

Fig. 5. The solid and dashed curves show the dependences of $\gamma_{c} / \gamma$ and $\gamma_{s} / \gamma$ on $a / b$ respectively for $\rho_{\mathrm{i}} / \rho_{\mathrm{e}}=10$.

(ii) The eigenfrequencies $\omega_{1 c, s}$ correspond to the two kink modes of the tube. They are given by Eq. (60). The kink mode with the frequency $\omega_{1 c}$ is polarized along the large axis, and with the frequency $\omega_{1 s}$ along the small axis of the elliptic cross-section.

(iii) The ratio of the mode decrements to the mode frequencies are of the order of $\ell / a$, where $\ell$ is the characteristic thickness of the inhomogeneous layer at the tube boundary. The decrements are given by Eqs. (78) and (79). For moderate values of the ratio of the large, $a$, and small, $b$, half-axis of the elliptic tube cross-section $(a / b \lesssim 2)$ the decrements of the two kink modes do not differ very much from the decrement of the kink mode of a tube with the circular cross-section.

The results obtained in this paper may be important for interpretation of the observed coronal loop oscillations. When analyzing the results of the observations it is assumed that an oscillation of a coronal loop is a damped harmonic oscillation. Then the best fit is used to determine its amplitude, phase and decrement.

The results obtained in this paper imply that coronal loop oscillations may be superpositions of two damped harmonic oscillations with different frequencies rather than a single damped harmonic oscillation. It would be rather interesting to analyze observations of coronal loop oscillations assuming that these oscillations are superpositions of two harmonic oscillations.

\section{Appendix A}

In this Appendix we calculate the asymptotic expressions for $\operatorname{Fek}_{n}(z, \theta)$ and $\operatorname{Gek}_{n}(z, \theta)$ valid when $\theta<0$ and $|\theta| \ll 1$. We use the expansions of $\operatorname{ce}_{n}(z, \theta), \mathrm{se}_{n}(s, \theta)$, and the modified Mathieu functions $\operatorname{Fey}_{n}(z, \theta)$ and $\operatorname{Gey}_{n}(z, \theta)$ in series of products of Bessel functions, the relations (30), and the expressions of $\operatorname{Fek}_{n}(z, \theta)$ and $\operatorname{Gek}_{n}(z, \theta)$ in terms of $\operatorname{Ce}_{n}(z, \theta), \operatorname{Se}_{n}(s, \theta)$, $\operatorname{Fey}_{n}(z, \theta)$ and $\operatorname{Gey}_{n}(z, \theta)$ (Bateman, Sects. 16.5 and 16.6). In addition, we use the relations

$$
J_{n}(i z)=i^{n} I_{n}(z), \quad H_{n}^{(1)}=\frac{2}{\pi} i^{-n-1} K_{n}(z),
$$

where $J_{n}$ is the Bessel function, $I_{n}$ the modified Bessel function, $H_{n}^{(1)}$ the Hankel function, and $K_{n}$ the modified Bessel function of the third kind (McDonald function). As a result, we obtain the following expressions valid for $\theta<0$ :

$$
\begin{aligned}
\operatorname{Fek}_{2 m}(z, \theta)= & \frac{p_{2 m}}{\pi A_{0}^{2 m}} \sum_{r=0}^{\infty}(-1)^{r} A_{2 r}^{2 m} I_{r}\left(|\theta|^{1 / 2} \mathrm{e}^{-s}\right) K_{r}\left(|\theta|^{1 / 2} \mathrm{e}^{s}\right) \\
\operatorname{Fek}_{2 m+1}(z, \theta)= & \frac{p_{2 m+1}}{\pi A_{1}^{2 m+1}} \sum_{r=0}^{\infty}(-1)^{r} A_{2 r+1}^{2 m+1} \\
& \times\left[I_{r}\left(|\theta|^{1 / 2} \mathrm{e}^{-s}\right) K_{r+1}\left(|\theta|^{1 / 2} \mathrm{e}^{s}\right)\right. \\
& \left.-I_{r+1}\left(|\theta|^{1 / 2} \mathrm{e}^{-s}\right) K_{r}\left(|\theta|^{1 / 2} \mathrm{e}^{s}\right)\right]
\end{aligned}
$$

$$
\begin{aligned}
\operatorname{Gek}_{2 m+1}(z, \theta)= & \frac{q_{2 m+1}}{\pi B_{1}^{2 m+1}} \sum_{r=0}^{\infty}(-1)^{r} B_{2 r+1}^{2 m+1} \\
& \times\left[I_{r}\left(|\theta|^{1 / 2} \mathrm{e}^{-s}\right) K_{r+1}\left(|\theta|^{1 / 2} \mathrm{e}^{s}\right)\right. \\
& \left.+I_{r+1}\left(|\theta|^{1 / 2} \mathrm{e}^{-s}\right) K_{r}\left(|\theta|^{1 / 2} \mathrm{e}^{s}\right)\right],
\end{aligned}
$$

$$
\begin{aligned}
\operatorname{Gek}_{2 m+2}(z, \theta)= & \frac{q_{2 m+2}}{\pi B_{2}^{2 m+2}} \sum_{r=0}^{\infty}(-1)^{r} B_{2 r+2}^{2 m+2} \\
& \times\left[I_{r}\left(|\theta|^{1 / 2} \mathrm{e}^{-s}\right) K_{r+2}\left(|\theta|^{1 / 2} \mathrm{e}^{s}\right)\right. \\
& \left.-I_{r+2}\left(|\theta|^{1 / 2} \mathrm{e}^{-s}\right) K_{r}\left(|\theta|^{1 / 2} \mathrm{e}^{s}\right)\right],
\end{aligned}
$$

where $m=0,1,2, \ldots$, and

$A_{0}^{2 m} p_{2 m}=\operatorname{ce}_{2 m}(0) \mathrm{ce}_{2 m}(\pi / 2)$,

$|\theta|^{1 / 2} A_{1}^{2 m+1} p_{2 m+1}=i \operatorname{ce}_{2 m+1}(0) \mathrm{ce}_{2 m+1}^{\prime}(\pi / 2)$,

$|\theta|^{1 / 2} B_{1}^{2 m+1} q_{2 m+1}=-i \mathrm{se}_{2 m+1}^{\prime}(0) \mathrm{se}_{2 m+1}(\pi / 2)$,

$\theta B_{2}^{2 m+2} q_{2 m+2}=\operatorname{se}_{2 m+2}^{\prime}(0) \operatorname{se}_{2 m+2}^{\prime}(\pi / 2)$.

Using Eq. (48) together with the asymptotics for the modified Bessel functions valid for small $z$ (Abramowitz \& Stegun 1964),

$I_{n}(z) \sim \frac{z^{n}}{2^{n} n !}, \quad K_{0}(z) \sim-\ln \frac{z}{2}-\gamma, \quad K_{n+1} \sim \frac{2^{n} n !}{z^{n+1}}$,

where $n=0,1,2, \ldots$ and $\gamma \approx 0.577$ is Euler's constant, we obtain from (A.2)-(A.5) the following asymptotics for the modified Mathieu functions valid for $|\theta|^{1 / 2} \mathrm{e}^{s} \ll 1$ :

$$
\begin{aligned}
& \operatorname{Fek}_{0}(z, \theta) \sim-\frac{p_{0}}{2 \pi}(\ln |\theta|+2 z+2 \gamma-2 \ln 2), \\
& \operatorname{Fek}_{2 m+1}(z, \theta) \sim \frac{(-1)^{m} p_{2 m+1}}{\pi|\theta|^{1 / 2} A_{1}^{2 m+1}} \mathrm{e}^{-(2 m+1) z}, \\
& \operatorname{Fek}_{2 m+2}(z, \theta) \sim \frac{(-1)^{m} p_{2 m+2}}{2 \pi(m+1) A_{0}^{2 m+2}} \mathrm{e}^{-(2 m+2) z}, \\
& \operatorname{Gek}_{2 m+1}(z, \theta) \sim \frac{(-1)^{m} q_{2 m+1}}{\pi|\theta|^{1 / 2} B_{1}^{2 m+1}} \mathrm{e}^{-(2 m+1) z}, \\
& \operatorname{Gek}_{2 m+2}(z, \theta) \sim \frac{2(m+1)(-1)^{m+1} q_{2 m+2}}{\pi \theta B_{2}^{2 m+2}} \mathrm{e}^{-(2 m+2) z},
\end{aligned}
$$

where $m=0,1,2, \ldots$ 


\section{References}

Abramowitz, M., \& Stegun, I. A. 1964, Handbook of Mathematical Functions (National Bureau of Standards, Washington DC)

Aschwanden, M. J., Fletcher, L., Schrijver, C. J., et al. 1999, ApJ, 520, 880

Aschwanden, M. J., De Pontieu, B., Schrijver, C. J., et al. 2002, Sol. Phys., 206, 99

Bateman, H. 1955, Higher Transcendental Functions, (New York: McGraw-Hill), vol. III

Cally, P. S. 1986, Sol. Phys., 103, 277

Defouw, R. J. 1976, ApJ, 209, 266

Edwin, P. M., \& Roberts, B. 1982, Sol. Phys., 76, 239

Edwin, P. M., \& Roberts, B. 1983, Sol. Phys., 88, 179

Goossens, M. 1991, MHD waves and wave heating in non-uniform plasmas, in Advances in Solar System Magnetohydrodynamics, ed. E. R. Priest, \& A. W. Hood (Cambridge: Cambridge Univ. Press), 135

Goossens, M., \& Ruderman, M. S. 1995, Phys. Scripta, T60, 171

Goossens, M., Ruderman, M. S., \& Hollweg, J. V. 1995, Sol. Phys., 157,75
Goossens, M., Andries, J., \& Aschwanden, M. J. 2002, A\&A, 394, L39

Hollweg, J. V. 1987, ApJ, 312, 880

Hollweg, J. V., \& Yang, G. 1988, J. Geophys. Res., 93, 5423

Korn, G. A., \& Korn, T. M. 1961, Mathematical Handbook for Scientists and Engineers (New York: McGraw-Hill)

Nakariakov, V. M., Ofman, L., Deluca, E. E., et al. 1999, Nature, 285, 862

Ofman, L., \& Aschwanden, M. J. 2002, ApJ, 576, L153

Roberts, B. 2000, Sol. Phys., 193, 139

Ruderman, M. S., \& Roberts, B. 2002, ApJ, 577, 475

Ruderman, M. S., \& Wright, A. N. 2000, Phys. Plasmas, 7, 3515

Ruderman, M. S., Tirry, W. J., \& Goossens, M. 1995, J. Plasma Phys., 54,129

Ryutov, D. D., \& Ryutova, M. P. 1976, Sov. Phys - JETP, 43, 491

Sakurai, T., Goossens, M., \& Hollweg, J. V. 1991, Sol. Phys., 133, 227

Schrijver, C. J., \& Brown, D. S. 2000, ApJ, 537, L69

Schrijver, C. J., Aschwanden, M. J., \& Title, A. M. 2002, Sol. Phys., 206, 69

Spruit, H. C. 1982, Sol. Phys., 75, 3

Tirry, W. J., \& Goossens, M. 1996, ApJ, 471, 501 